\title{
TU/e EmonONEN

\section{The interplay between shell effects and electron correlations in quantum dots}

Citation for published version (APA):

Harting, J. D. R., Mülken, O., \& Borrmann, P. (2000). The interplay between shell effects and electron correlations in quantum dots. Physical Review B, 62(15), 10207-10211.

https://doi.org/10.1103/PhysRevB.62.10207

DOI:

10.1103/PhysRevB.62.10207

Document status and date:

Published: 01/01/2000

\section{Document Version:}

Publisher's PDF, also known as Version of Record (includes final page, issue and volume numbers)

\section{Please check the document version of this publication:}

- A submitted manuscript is the version of the article upon submission and before peer-review. There can be important differences between the submitted version and the official published version of record. People interested in the research are advised to contact the author for the final version of the publication, or visit the $\mathrm{DOI}$ to the publisher's website.

- The final author version and the galley proof are versions of the publication after peer review.

- The final published version features the final layout of the paper including the volume, issue and page numbers.

Link to publication

\section{General rights}

Copyright and moral rights for the publications made accessible in the public portal are retained by the authors and/or other copyright owners and it is a condition of accessing publications that users recognise and abide by the legal requirements associated with these rights.

- Users may download and print one copy of any publication from the public portal for the purpose of private study or research.

- You may not further distribute the material or use it for any profit-making activity or commercial gain

- You may freely distribute the URL identifying the publication in the public portal.

If the publication is distributed under the terms of Article 25fa of the Dutch Copyright Act, indicated by the "Taverne" license above, please follow below link for the End User Agreement:

www.tue.nl/taverne

Take down policy

If you believe that this document breaches copyright please contact us at:

openaccess@tue.nl

providing details and we will investigate your claim. 


\title{
Interplay between shell effects and electron correlations in quantum dots
}

\author{
Jens Harting, Oliver Mülken, and Peter Borrmann \\ Department of Physics, Carl von Ossietzky University Oldenburg, D-26111 Oldenburg, Germany
}

(Received 18 February 2000)

\begin{abstract}
We use the path integral Monte Carlo method to investigate the interplay between shell effects and electron correlations in single quantum dots with up to 12 electrons. By use of an energy estimator based on the hypervirial theorem of Hirschfelder we study the energy contributions of different interaction terms in detail. We discuss under which conditions the total spin of the electrons is given by Hund's rule, and the temperature dependence of the crystallization effects.
\end{abstract}

\section{INTRODUCTION}

The advances in nanofabrication of the last years opened the goal to build two-dimensional (2D) quantum dots (QD's) and quantum dot molecules (QDM's) — artificial mesoscopic semiconductor structures of selectable shape and size-as containers for a controllable fixed number of electrons. ${ }^{1,2}$ Recently, depending on the strength and shape of the effective confining potential, the formation of spin density waves (SDW's) (Refs. 3 and 4) and Wigner crystals ${ }^{3,5}$ in QD's and QDM's has been predicted by different groups with different theoretical approaches. Hirose and Wingreen ${ }^{6}$ argue that SDW's are reproducible artifacts of spin density functional calculations. For a 2D parabolic confining potential the accordance of the spin configuration with Hund's rule has been predicted by Koskinen, Manninen, and Reimann ${ }^{4}$ and questioned by Yannouleas and Landman. ${ }^{3}$ All these effects are governed by the intriguing interplay between shell effects, the pure Coulomb repulsion, and the fermionic repulsion due to the Pauli exclusion principle and depend strongly on the values of the interaction parameters in the commonly assumed Hamiltonian for single QD's

$$
H=\sum_{i=1}^{N}\left(\frac{\mathbf{p}_{i}^{2}}{2 m^{*}}+\frac{m^{*} \omega_{0}^{2}}{2} \mathbf{x}_{i}^{2}\right)+\sum_{i<j=1}^{N} \frac{e^{2}}{\kappa\left|\mathbf{x}_{i}-\mathbf{x}_{j}\right|},
$$

where $\kappa$ is the dielectric constant, $m^{*}$ is the effective mass, and $\omega_{0}$ defines the strength of the confining potential.

Apart from the interesting physical questions that arise for quantum dots the reliable prediction of their properties is an ultimate test of modern methods in quantum chemistry. Due to the compared to atoms very shallow confining potential, long range electron interactions and correlations play an important role in QD's and QDM's. Therefore it is misleading to name them artificial atoms and molecules. Well established and very elaborate methods of quantum chemistry might fail in describing them properly. Hartree-Fock and spin density functional methods use single Slater determinants or sums of them to approximate the many-body wave function. In spin-density functional methods the approximation of the functional for the exchange correlation energy $4,7,8$ adds another source of uncertainty and systematic errors to this approach. The path integral Monte Carlo method (PIMC) used in this paper samples the full many-body wave function instead.

In contrast to density functional methods (DFT) with PIMC it is possible to study the temperature dependent prop- erties of QD's. The reason why PIMC is not yet a standard method of quantum chemistry is its numerical limitation due to the fermion sign problem. The rapidly increasing power of modern computers resizes this limitation. In Sec. II we briefly summarize our implementation of PIMC and comment on how to limit the numerical deficiencies due to the fermion sign problem.

We apply PIMC to calculate the electron density and twoparticle correlation functions for quantum dots with up to 12 electrons. To compare with various experimental studies as well as with other theoretical studies we use different dielectric constants $\kappa$ and strengths of the confining parabolic potentials. The calculated addition energies are in very good agreement with the experimental findings of Tarucha et al. ${ }^{2}$

For $N=6$ we investigate the temperature dependence of the Wigner crystallization (WC).

\section{NUMERICAL METHOD}

For a system of $N$ electrons with position eigenket $\left|\vec{x}_{i}, s_{i}\right\rangle$ ( $s_{i}= \pm \frac{1}{2}$ for spin-up and spin-down electrons) in an external potential the Feynman path integral can be written as ${ }^{9-11}$

$$
\begin{aligned}
Z= & \int\left[\prod_{\gamma=1}^{M} \prod_{i=1}^{N} \mathrm{~d} \vec{x}_{i}(\gamma)\right] \prod_{\delta=1}^{M} \operatorname{det}[A(\delta, \delta+1)] \\
& \times \exp \left(-\frac{\beta}{M} \sum_{\alpha=1}^{M} V\left[\vec{x}_{1}(\alpha), \ldots, \vec{x}_{N}(\alpha)\right]\right)+O\left(\frac{\beta^{3}}{M^{2}}\right),
\end{aligned}
$$

with

$$
\begin{aligned}
& {[A(\alpha, \alpha+1)]_{i, j}} \\
& \quad= \begin{cases}\left\langle\vec{x}_{i}(\alpha)\left|\exp \left(-\frac{\beta}{M} \frac{\mathbf{p}^{2}}{2 m}\right)\right| \vec{x}_{j}(\alpha+1)\right\rangle, & s_{i}=s_{j} \\
0, & s_{j} \neq s_{j}\end{cases}
\end{aligned}
$$

and the boundary condition $\vec{x}_{j}(M+1)=\vec{x}_{j}(1) . M$ is the number of so-called timeslices of the Feynman paths. In the limit $M \rightarrow \infty$ Eq. (2) becomes exact. For quantum dots the space dimension is $d=2$ and the $(2 N M)$-dimensional integral given in Eq. (2) can be evaluated by standard Metropolis 
Monte Carlo techniques. Due to the determinant the integrand is not always positive and the expectation value of an observable $X(\mathbf{x})$ depending only on position operators has to be calculated using

$$
\langle X\rangle=\frac{\sum_{g=1}^{G} X_{g} \operatorname{sgn}\left(W_{g}\right)}{\sum_{g=1}^{G} \operatorname{sgn}\left(W_{g}\right)},
$$

where $X_{g}$ is the value of the observable $X$ and $W_{g}$ is the value of the integrand in Eq. (2) in the $g$ th Monte Carlo step. Equation (4) reveals a severe problem connected with the path integral for fermions which is commonly denoted as the fermion sign problem (see, e.g., Refs. 12-14). It can be shown that the ratio between integrands with positive sign $\left(W^{+}\right)$and negative sign $\left(W^{-}\right)$is approximately given by ${ }^{14,15}$

$$
\frac{W^{+}-W^{-}}{W^{+}+W^{-}} \sim \exp \left[-\beta\left(E_{F}-E_{B}\right)\right],
$$

where $E_{F}$ and $E_{B}$ are the ground state energies of the Fermi system and the corresponding Bose system. It is now obvious that the statistical error in Eq. (4) grows rapidly for small temperatures $T$. Moreover the energy difference $\left(E_{F}-E_{B}\right)$ will grow with increasing system size causing an increase of the statistical error.

Within PIMC the calculation of the kinetic energy expectation value is another critical task. This is merely due to the fact that the Monte Carlo calculation is usually done in position space and that the discretization of the paths allows a number of different approaches to calculate the expectation value of a momentum dependent operator. A number of various different energy estimators has been discussed in the past. ${ }^{16-18}$

To avoid these difficulties we developed a procedure which allows the calculation of all energy expectation values from the knowledge of the pair correlation functions

$$
\Gamma_{i, j}(r)=\left\langle\delta\left(r-\left|\vec{x}_{i}-\vec{x}_{j}\right|\right)\right\rangle
$$

and the radial density functions per electron

$$
\rho_{i}(r)=\frac{1}{2 \pi r}\left\langle\delta\left(r-\left|\vec{x}_{i}\right|\right)\right\rangle=\frac{1}{2 \pi r} \varrho(r),
$$

where $\varrho$ is the probability of finding electron $i$ in distance $r$ from the center.

Due to the particle symmetry we have

$$
\Gamma_{i, j}(r)= \begin{cases}\Gamma^{\uparrow \uparrow}(r), & s_{i}=s_{j}=+\frac{1}{2} \\ \Gamma^{\downarrow \downarrow}(r), & s_{i}=s_{j}=-\frac{1}{2} \\ \Gamma^{\uparrow \downarrow}(r), & s_{i} \neq s_{j}\end{cases}
$$

and

$$
\rho_{i}(r)= \begin{cases}\rho^{\uparrow}(r), & s_{i}=+\frac{1}{2} \\ \rho^{\downarrow}(r), & s_{i}=-\frac{1}{2} .\end{cases}
$$

Utilizing the hypervirial theorem of Hirschfelder ${ }^{19}$ the energy can be written as a sum of ten parts ${ }^{20}$

$$
\begin{aligned}
E= & E_{\mathrm{kin}}^{\uparrow}+E_{\mathrm{kin}}^{\downarrow}+E_{\mathrm{kin}}^{\uparrow \uparrow}+E_{\mathrm{kin}}^{\downarrow \downarrow}+E_{\mathrm{kin}}^{\uparrow \downarrow}+E_{\mathrm{pot}}^{\uparrow}+E_{\mathrm{pot}}^{\downarrow}+E_{\mathrm{pot}}^{\uparrow \uparrow}+E_{\mathrm{pot}}^{\downarrow \downarrow}+E_{\mathrm{pot}}^{\uparrow \downarrow}=\frac{N^{\uparrow}}{2} \int_{0}^{\infty} d r \varrho^{\uparrow}(r) r \partial_{r} V_{1}(r)+\frac{N^{\downarrow}}{2} \int_{0}^{\infty} d r \varrho^{\downarrow}(r) r \partial_{r} V_{1}(r) \\
& +\frac{N^{\uparrow}\left(N^{\uparrow}-1\right)}{4} \int_{0}^{\infty} d r \Gamma^{\uparrow \uparrow}(r) r \frac{\partial V_{2}(r)}{\partial r}+\frac{N^{\downarrow}\left(N^{\downarrow}-1\right)}{4} \int_{0}^{\infty} d r \Gamma^{\downarrow \downarrow}(r) r \frac{\partial V_{2}(r)}{\partial r}+\frac{N^{\downarrow} N^{\uparrow}}{2} \int_{0}^{\infty} d r \Gamma^{\uparrow \downarrow}(r) r \frac{\partial V_{2}(r)}{\partial r} \\
& +\frac{N^{\uparrow}}{2} \int_{0}^{\infty} d r \varrho^{\uparrow}(r) V_{1}(r)+\frac{N^{\downarrow}}{2} \int_{0}^{\infty} d r \varrho^{\downarrow}(r) V_{1}(r)+\frac{N^{\uparrow}\left(N^{\uparrow}-1\right)}{2} \int d r \Gamma^{\uparrow \uparrow}(r) V_{2}(r) \\
& +\frac{N^{\downarrow}\left(N^{\downarrow}-1\right)}{2} \int d r \Gamma^{\downarrow \downarrow}(r) V_{2}(r)+N^{\uparrow} N^{\downarrow} \int d r \Gamma^{\uparrow \downarrow}(r) V_{2}(r) .
\end{aligned}
$$

While in density functional approaches the calculation of the kinetic energy and the exchange correlation energy is a major topic and subject to permanent discussion, within the path integral approach these energies are included in a natural way.

However, the systematic error arising from the limited number of timeslices $M$ and the statistical error of the Monte Carlo calculation have to be controlled carefully. We checked our algorithm extensively using eight noninteracting fermions in a parabolic trap as a test system. We found that at low temperatures where the ratio of signs is around 0.99, convergence can only be achieved obeying the following rules: (1) The determinants have to be calculated very accurately using a more costly algorithm with pivoting. (2) The completely uncorrelated generation of the Monte Carlo steps is essential, i.e., the coordinate to be moved should be chosen randomly. Moving the particle coordinates using always the same sequence produces inaccurate results. (3) A good random number generator with a completely uncorrelated sequence in all significant bits of a 64 bit real number should be applied. We therefore developed a 53 bit random number of Marsaglia-Zaman type ${ }^{21}$ instead of using one of the standard 24 or 32 bit random number generators coming with standard system libraries. (4) Further, to improve the conver- 


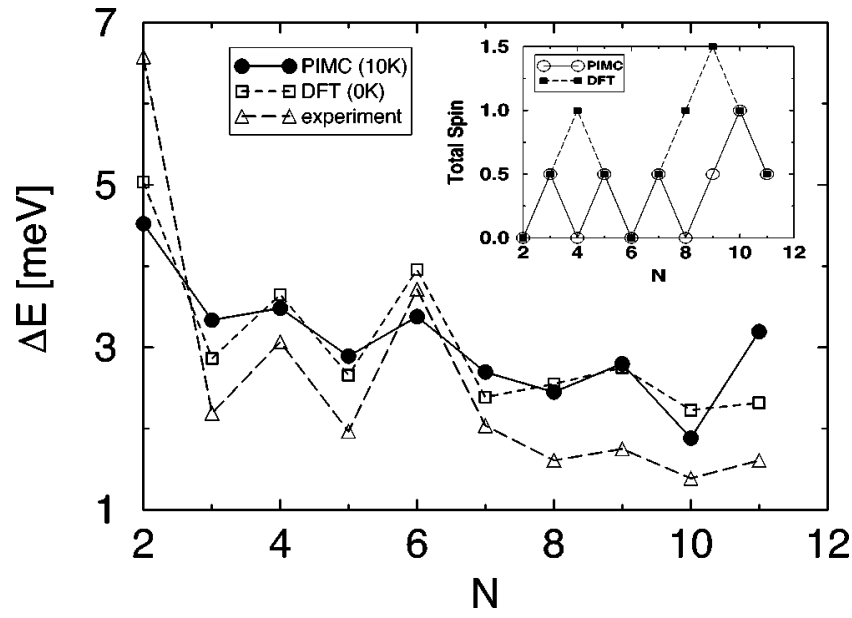

FIG. 1. Addition energies for quantum dots with up to 11 electrons. The circles indicate the results from our path integral calculations at $10 \mathrm{~K}$, the squares are results of spin density functional calculations of Hirose and Wingreen, and the triangles are the experimental results of Tarucha et al. The error bars for PIMC would be of the size of the solid circles and are therefore omitted.

gence a number of different Monte Carlo steps can be applied, i.e., moving single time slices, moving complete particle paths and parts of a path. Our Fortran code is completely parallelized using MPI and Lapack.

\section{RESULTS}

To compare our PIMC calculations to experimental data we calculated the addition energies

$$
\Delta E=E_{N+1}-2 E_{N}+E_{N-1}
$$

of a QD with up to 11 electrons using the material constants $m^{*}=0.067 m$ and $\kappa=12.9$ for GaAs as given by Hirose and Wingreen. ${ }^{6}$ It is assumed that these parameters mimic the experimental setup of Tarucha et al..$^{2}$ reasonably well. The strength of the harmonic potential is fixed at $\hbar \omega_{0}$ $=3.0 \mathrm{meV}$. The resulting effective atomic units are $E_{H}^{*}$ $=10.955 \mathrm{meV}$ for the Hartree energy and $a_{0}^{*}$ $=10.1886 \mathrm{~nm}$ for the Bohr radius. The Boltzmann constant is $k_{B}=7.8661 \times 10^{-3} E_{H}^{*} / \mathrm{K}$.

We performed PIMC simulations for quantum dots with different spin configurations at a fixed temperature of $10 \mathrm{~K}$. Due to the fermion sign problem the number of Monte Carlo steps necessary to push the statistical error of the total energy, which has been calculated properly from 25 uncorrelated subsequences of $\mathrm{MC}$ steps, into the range of $0.1 \%$ is extremely high. The number of Monte Carlo steps ranged between 2.5 billion steps per particle coordinate for $N \leqslant 6$ and about 10 billion steps for $N=12$. Figure 1 displays the addition energies for quantum dots with up to 11 electrons. The circles indicate the results from our path integral calculations at $10 \mathrm{~K}$, the squares are results of spin density functional calculations of Hirose and Wingreen, ${ }^{6}$ and the triangles are the experimental results of Tarucha et al. ${ }^{2}$ Both theoretical calculations reproduce the general $N$ dependence of the addition energies in great detail. Tarucha $e t$ al. give an estimate of the electron temperature in their experiments of $T=0.2 \mathrm{~K}$. For computational reasons our PIMC calculations are performed at $10 \mathrm{~K}$ and it cannot be expected that the
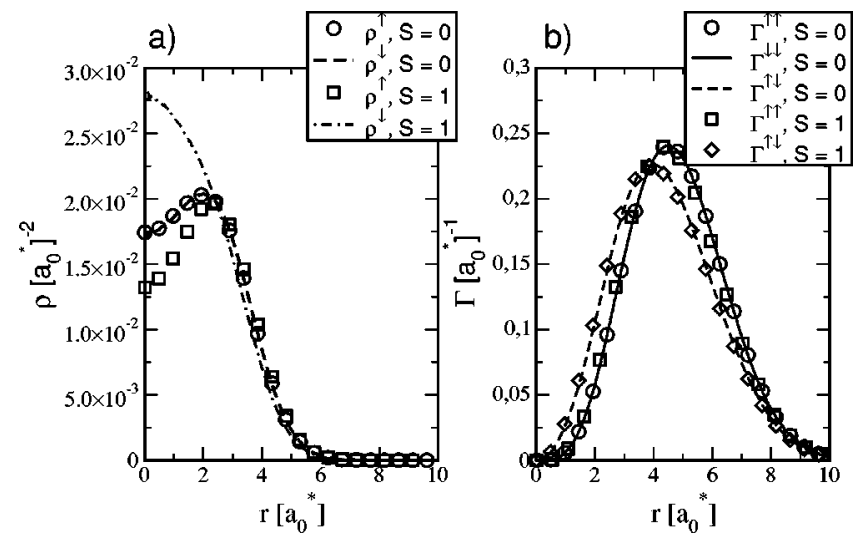

FIG. 2. (a) Radial density per electron and (b) pair correlation functions for four electrons and total spins $S=0$ and $S=1$. The material constants are $\kappa=12.9$ and $\hbar \omega=3.0 \mathrm{meV}$.

absolute energy values agree as well as the $0 \mathrm{~K}$ DFT calculations with the experimental results. However, it should be noted that PIMC correctly predicts the drop in the addition energy from $N=7$ to $N=8$ while the DFT calculations fail at this point.

The inset in Fig. 1 displays the total spins of the spin configurations with lowest energy as found in DFT and PIMC at $10 \mathrm{~K}$. In DFT calculations $(0 \mathrm{~K})$ the spin configuration of the ground state is determined by Hund's rule for up to 22 electrons. In contrast, in our PIMC calculation at 10 $\mathrm{K}$ the total spin is not always in accordance with Hund's rule. For $N=4$ we checked the temperature dependence of the spin configuration. At $5 \mathrm{~K}$ the energy of the spin 0 configuration is $0.01 E_{H}^{*}$ higher than the spin 1 energy indicating a temperature dependence of the favored spin configuration.

As an important fact we note that the $N$ dependence of the addition energies is not affected by the actual spin configuration. The situation is quite similar to that in transition metal clusters with extreme small energy differences between states with significantly different magnetic moments. ${ }^{22}$

As can be inferred from Fig. 2(a) the radial spin densities are significantly different for both spin configurations. The total potential energy for the spin 1 configuration is about $0.07 \mathrm{meV}$ lower than that of the spin 0 configuration. At 10 $\mathrm{K}$ this is overcompensated by an $0.27 \mathrm{meV}$ higher kinetic energy (see Table I). Although the kinetic and potential energies for different total spins significantly differ, the total energies are almost equal. Similar situations are found for larger $N$.

For convenience and easy comparison we determined the value of the dimensionless density parameter $r_{s}$, which is sometimes used to characterize quantum dots (see, e.g., Ref. 5) to be $r_{s}=4.19$ for $N=4$.

The energies given in Table I correspond to the integrals in Eq. (10). The total kinetic energy which is the sum of all $E_{\mathrm{kin}}^{x}$ terms is always positive while some of the addends might be negative. Table I reveals that larger total spins result in larger kinetic energies. The total potential energy is almost unchanged, the larger contribution from the trap potential is compensated by a smaller contribution from the Coulomb repulsion. We note that the ratio $E_{\mathrm{W}}$ between the kinetic energy and the total energy is considerably larger for $N=4$ than for $N=9$ reflecting the looser binding of the smaller system. 
TABLE I. Kinetic and potential energies as well as $E_{\mathrm{W}}=E_{\mathrm{kin}} / E_{\mathrm{tot}}$ in $\mathrm{meV}$ for different electron configurations at $10 \mathrm{~K}$ (the numbers in parentheses are the single particle energies).

\begin{tabular}{lcccc}
\hline \hline & $N^{\uparrow}=2, N^{\downarrow}=2$ & $N^{\uparrow}=3, N^{\downarrow}=1$ & $N^{\uparrow}=5, N^{\downarrow}=4$ & $N^{\uparrow}=6, N^{\downarrow}=3$ \\
\hline$E_{\text {tot }}$ & 40.83 & 41.03 & 169.25 & 169.82 \\
$E_{\text {kin }}$ & 7.33 & 7.60 & 18.97 & 19.57 \\
$E_{\text {pot }}$ & 33.50 & 33.43 & 150.28 & 150.26 \\
$E_{\mathrm{W}}$ & 0.18 & 0.19 & 0.11 & 0.12 \\
\hline$E_{\text {kin }}^{\uparrow}$ & $8.03(8.03)$ & $12.66(8.44)$ & $35.44(14.18)$ & $43.74(14.58)$ \\
$E_{\text {kin }}^{\downarrow}$ & $8.03(8.03)$ & $3.55(7.10)$ & $27.30(13.65)$ & $19.39(12.93)$ \\
$E_{\text {kin }}^{\uparrow \uparrow}$ & $-1.31(-2.62)$ & $-3.97(-2.65)$ & $-11.20(-2.24)$ & $-16.87(-2.25)$ \\
$E_{\text {kin }}^{\uparrow \downarrow}$ & $-6.11(-3.05)$ & $-4.64(-3.09)$ & $-25.84(-2.58)$ & $-23.31(-2.59)$ \\
$E_{\text {kin }}^{\downarrow \downarrow}$ & $-1.31(-2.62)$ & $0.00(0.00)$ & $-6.72(-2.24)$ & $-3.39(-2.26)$ \\
\hline$E_{\text {pot }}$ & 33.50 & 33.43 & 150.28 & 150.26 \\
$E_{\text {pot }}^{\uparrow}$ & $8.03(4.01)$ & $12.66(4.22)$ & $35.44(7.09)$ & $43.74(7.30)$ \\
$E_{\text {pot }}^{\downarrow}$ & $8.03(4.01)$ & $3.55(3.55)$ & $27.30(6.82)$ & $19.39(6.46)$ \\
$E_{\text {pot }}^{\uparrow \uparrow}$ & $2.62(2.62)$ & $7.94(2.65)$ & $22.40(2.24)$ & $33.73(2.25)$ \\
$E_{\text {pot }}^{\uparrow \downarrow}$ & $12.21(3.05)$ & $9.28(3.09)$ & $51.69(2.58)$ & $46.62(2.59)$ \\
$E_{\text {pot }}^{\downarrow \downarrow}$ & $2.62(2.62)$ & $0.00(0.00)$ & $13.44(2.24)$ & $6.78(2.26)$ \\
\hline \hline
\end{tabular}

Next we consider the dependence of the Wigner crystallization on the temperature and the choice of the material constants. The localization of the electrons in space is commonly referred to as Wigner crystallization. For quantum dots the occurrence of well separated humps in the radial electron density and the pair correlation functions has been interpreted as WC. However, it is a nontrivial task to find a general parameter identifying if an electron system is crystallized or not. From a solid state physics point of view the electrons should have a low mobility, i.e., a small kinetic energy, and should not interchange their lattice positions. For fermions the localization of single electrons does not make any sense, and, as stated above, even the decomposition of the many-body wave function in sums of determinants of single particle wave functions is probably a too rough approximation for QD's. These facts limit the analogies between crystallization in solids and electron systems and make the term crystallization itself somehow misleading. We therefore view Wigner crystals as states of the many-body wave function with a relatively low kinetic energy.

First we consider the strength of the Wigner crystallization depending on the choice of the interaction parameters. Figure 3 displays the radial densities and pair correlation functions for six electrons with $S=0, \hbar \omega=5 \mathrm{meV}$, and $\kappa$ $=3.0,6.0$, and 12.9. Of course, for stronger electron repulsions $($ small $\kappa)$ the electron distributions are broadened. The qualitative picture of the distributions is merely the same. For all $\kappa$ shell effects indicated by off-center maximums of the radial density occur. However, only for $\kappa=3$ and 6 we observe a maximum at $r=0$. From our point of view it cannot definitely be decided from this figure if a system is Wigner crystallized or not. As a parameter reflecting the strength of the WC we employ the ratios between the kinetic and the total energies $E_{\mathrm{W}}=E_{\mathrm{kin}} / E_{\text {tot }}$ which are $0.07,0.10$, and 0.14 for $\kappa=3.0,6.0$, and 12.9 . Although the radial distribution function for $\kappa=12.9$ is quite narrow, the relative mobility for the electrons indicated by $E_{\mathrm{W}}$ is twice as large as for $\kappa=3$. The underlying physical process can be under- stood intuitively. Due to the stronger electron-electron repulsions the electrons are fixed in an energetical favorable geometric configuration and as a consequence thereof the relative kinetic energy is reduced and the difference between the pair correlation functions of equal and opposite spin almost vanishes (see Fig. 3). It is an interesting and to our knowledge open question, if the crystallization of electrons can be viewed as a phase transition. We therefore consider next the temperature dependent properties of a quantum dot with $N=6, S=0, \kappa=3$, and $\hbar \omega=5 \mathrm{meV}$. The results for temperatures between 10 and $150 \mathrm{~K}$ are presented in Table II. Most notably $E_{\mathrm{W}}$ increases relatively smoothly from 0.07 at $10 \mathrm{~K}$ to 0.22 at $150 \mathrm{~K}$. Within our numerical accuracy the caloric curve does not show any evidence of a phase transition. The transition from a crystallized state to an electron fluid seems to be squashy. Of course, from our calculations we cannot exclude that a phase transition exists for larger $N$ a)

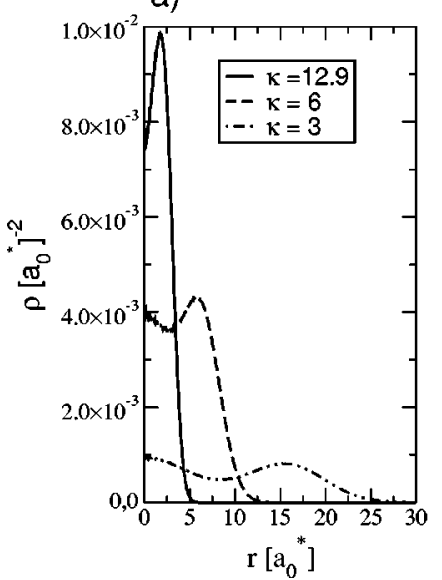

b)

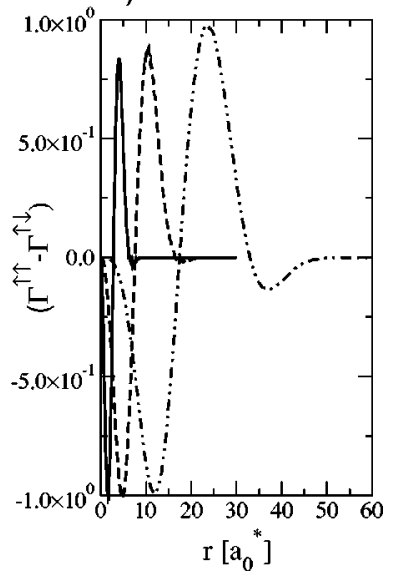

FIG. 3. Radial density per electron (left) and pair correlation functions (right) for $N=6, S=0, \hbar \omega_{0}=5 \mathrm{meV}$ and dielectric constants $\kappa=3.0,6.0$, and 12.9 at $10 \mathrm{~K}$. The radial density for $\kappa$ $=12.9$ is scaled by a factor of 3 and the pair correlation functions are scaled to have a maximum value of \pm 1 . 
TABLE II. Kinetic and potential energies for different temperatures and spin configuration $N^{\uparrow}=3, N^{\downarrow}=3$. The Hartree energy is $E_{H}^{*}=202.558 \mathrm{meV}, \kappa=3$, and $\hbar \omega_{0}=5.0 \mathrm{meV} . E_{\mathrm{W}}$ is the ratio between the kinetic and the total energy.

\begin{tabular}{ccccc}
\hline \hline$N^{\uparrow}=3, N^{\downarrow}=3$ & $E_{\mathrm{W}}$ & $E_{\text {kin }}(\mathrm{meV})$ & $E_{\text {pot }}(\mathrm{meV})$ & $E_{\text {tot }}(\mathrm{meV})$ \\
\hline$T=10 \mathrm{~K}$ & 0.07 & 17.70 & 246.53 & 264.32 \\
$T=30 \mathrm{~K}$ & 0.08 & 22.93 & 249.53 & 272.46 \\
$T=60 \mathrm{~K}$ & 0.12 & 35.39 & 257.67 & 293.06 \\
$T=90 \mathrm{~K}$ & 0.16 & 49.44 & 267.01 & 316.46 \\
$T=120 \mathrm{~K}$ & 0.19 & 64.10 & 276.84 & 340.93 \\
$T=150 \mathrm{~K}$ & 0.22 & 78.49 & 286.88 & 365.35 \\
\hline \hline
\end{tabular}

or different interaction parameters. Figure 4 displays the radial electron densities and the total electron pair correlation function for different temperatures. Up to $60 \mathrm{~K}$ the radial density shows clear geometric structure effects with two maximums while at $150 \mathrm{~K}$ only a smooth curve resembling a simple Gaussian remains.

\section{CONCLUSION}

In conclusion, we have found that despite of the notorious fermion sign problem PIMC is capable of answering interesting questions for strongly correlated electron systems like QD's and QDM's. For QD's PIMC reproduces correctly the experimental addition energies. Our temperature dependent calculations give new insights into the process of WC. For the two-dimensional QD's a ratio $E_{\mathrm{W}}=E_{\text {kin }} / E_{\text {tot }}$ below 0.1 seems to indicate WC both for $\kappa$ and temperature dependent calculations. However, regarding this aspect a more firm classification parameter, e.g., similar to the Lindemann criterion is desirable.

A comparision to other QMC methods seems to be in order here. Even our most complicated simulations took less than $2 \mathrm{~h}$ on a Cray T3E with 62 processors. Taking the advantages of modern computer power and optimized soft-
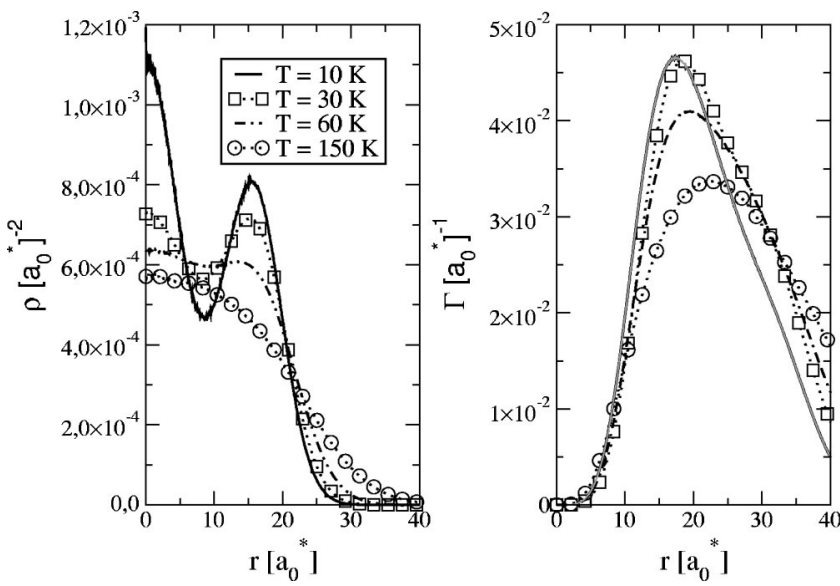

FIG. 4. Radial density (left) and pair correlation functions (right) for $N=6, S=0, \hbar \omega_{0}=5 \mathrm{meV}, \kappa=3.0$, and temperatures $T=10,30,60$, and $150 \mathrm{~K}$.

ware, the brute force PIMC applied here is able to produce very precise results. Different algorithms have been published to improve the performance of path integral methods. A recent one is the multilevel blocking method published by Mak et al. ${ }^{23}$ Simulations using this algorithm are expected to converge better than our direct treatment since the fermion sign problem is avoided partially. The published results of the treatment of quantum dots by Egger et al. ${ }^{5}$ do not confirm this expectation. Obviously the advantages of the new method are more than compensated by other numerical problems, maybe due to the energy estimator used. ${ }^{24}$ Nevertheless, a combination of the multilevel-blocking method and our technique might result in a very powerful tool.

\section{ACKNOWLEDGMENTS}

We wish to thank the Regionales Rechenzentrum Niedersachsen and the Konrad Zuse Institut Berlin for their excellent computer support and E. R. Hilf for stimulating discussions.
${ }^{1}$ N. Zhitenev, R. Ashoori, L. Pfeiffer, and K. West, Phys. Rev. Lett. 79, 2308 (1997).

${ }^{2}$ S. Tarucha, D. Austing, and T. Honda, Phys. Rev. Lett. 77, 3613 (1996).

${ }^{3}$ C. Yannouleas and U. Landman, Phys. Rev. Lett. 82, 5325 (1999).

${ }^{4}$ M. Koskinen, M. Manninen, and S. Reimann, Phys. Rev. Lett. 79, 1389 (1997).

${ }^{5}$ R. Egger, W. Häusler, C. Mak, and H. Grabert, Phys. Rev. Lett. 82, 3320 (1999).

${ }^{6}$ K. Hirose and N. Wingreen, Phys. Rev. B 59, 4604 (1999).

${ }^{7}$ D. Pfannkuche, V. Gudmundsson, and P. Maksym, Phys. Rev. B 47, 2244 (1993)

${ }^{8}$ R. Pino, Phys. Rev. B 58, 4644 (1998).

${ }^{9}$ M. Takahashi and M. Imada, J. Phys. Soc. Jpn. 53, 936 (1984).

${ }^{10}$ M. Takahashi and M. Imada, J. Phys. Soc. Jpn. 53, 3765 (1984).

${ }^{11}$ P. Borrmann and E. Hilf, Z. Phys. D: At., Mol. Clusters 26, S350 (1993).
${ }^{12}$ D. Ceperley, Phys. Rev. Lett. 69, 331 (1992).

${ }^{13}$ A. Lyubartsev and P. Vorontsov-Velayaminov, Phys. Rev. A 48, 4075 (1993).

${ }^{14}$ I. Morgenstern, Z. Phys. B: Condens. Matter 77, 267 (1989).

${ }^{15}$ W.H. Newman and A. Kuki, J. Chem. Phys. 96, 356 (1992).

${ }^{16}$ J. Cao and B. Berne, J. Chem. Phys. 91, 6359 (1989).

${ }^{17}$ A. Giansanti and G. Jacucci, J. Chem. Phys. 89, 7454 (1988).

${ }^{18}$ H. Kono, A. Takasaka, and S. Lin, J. Chem. Phys. 88, 6390 (1988).

${ }^{19}$ J.O. Hirschfelder, J. Chem. Phys. 33, 1462 (1960).

${ }^{20}$ H. Heinze, P. Borrmann, H. Stamerjohanns, and E. Hilf, Z. Phys. D: At., Mol. Clusters 40, 190 (1997).

${ }^{21}$ P. Borrmann (unpublished).

${ }^{22}$ K. Lee and J. Callaway, Phys. Rev. B 48, 15358 (1993).

${ }^{23}$ C. Mak, R. Egger, and H. Weber-Gottschick, Phys. Rev. Lett. 81, 4533 (1998).

${ }^{24}$ D. Ceperley, Phys. Rev. Lett. 69, 331 (1992). 